\title{
The effect of hyoscine butylbromide in shortening the first stage of labor: A double blind, randomized, controlled, clinical trial
}

This article was published in the following Dove Press journal:

Therapeutics and Clinical Risk Management

7 December 2011

Number of times this article has been viewed

\author{
Nourah H Al Qahtani' \\ Fatma Al Hajeri² \\ 'MRCOG, College of Medicine, \\ Department of Obstetrics and \\ Gynecology in King Fahad University \\ Hospital, University of Dammam, \\ Saudi Arabia; ${ }^{2}$ Department of \\ Obstetrics and Gynecology in King \\ Fahad University Hospital, \\ Saudi Arabia
}

Background and objectives: Hyoscine butylbromide (HBB) is widely used in labor rooms. There have been many studies on the use of HBB during labor with conflicting results, involving both primiparous and multiparous women. The aim of this trial was to study the efficacy of HBB for shortening the first stage of labor in primiparous women.

Methods: The study was a randomized, double blind, controlled trial. Ninety-seven primigravid term pregnant women in spontaneous labor received either hyoscine butylbromide or a placebo intramuscularly once the women entered the active phase of labor. The primary outcome measured was the duration of the first stage of labor. Secondary outcomes were the duration of the second and third stages of labor, blood loss at delivery, rate of cesarean section, and Apgar scores for the neonates.

Results: A total of 97 women yielded data for analysis. Of these, 45 women received the placebo and 52 received HBB. The mean duration of the first stage in the control group was 215 minutes, compared with 165 minutes in the study group, representing a decrease of $23.3 \%$ $(P=0.001)$. There were no significant changes in the duration of the second $(P=0.063)$ or third $(P=0.0418)$ stages of labor, and no significant differences in blood loss or Apgar scores. There was a slightly higher (but statistically insignificant) rate of instrumental delivery in the control group, but no difference in the Cesarean section rate. There was a $60 \%$ reduction in opioid analgesic use in the HBB group.

Conclusion: HBB is effective in significantly reducing the duration of the first stage of labor, and is not associated with any apparent adverse maternal or neonatal outcomes.

Keywords: hyoscine butylbromide, first stage, labor, duration, augmentation

\section{Introduction}

The management of normal labor is both an art and a science. For decades, health providers have worked to manage labor actively and safely, with the goal of shortening the duration of painful labor. Reduction of Cesarean sections and other fetal and maternal complications is also an important aspect of labor management.

The safety of active management of labor has been demonstrated by several prospective randomized clinical trials involving over 3000 women. ${ }^{1}$ A shorter duration of labor from admission to delivery has also been consistently reported in numerous studies of women treated with the active management protocol. ${ }^{1-3}$

Hyoscine butylbromide (HBB) belongs to the parasympatholytic group of drugs and is a semisynthetic derivative of scopolamine. It is an effective antispasmodic drug without the untoward side effects of atropine. HBB is a quaternary ammonium compound and has peripheral anticholinergic action, but no central action as it does 
not cross the blood-brain barrier. HBB acts primarily by blocking the transmission of neural impulses in the intraneural parasympathetic ganglia of abdominal organs, apparently inhibiting cholinergic transmission in the synapses of the abdominal and pelvic parasympathetic ganglia, thus relieving spasms in the smooth muscles of gastrointestinal, biliary, urinary tract, and female genital organs, especially the cervico-uterine plexus, thus aiding cervical dilatation. ${ }^{4,5}$

Spasmolytic drugs are frequently employed in delivery rooms to overcome cervical spasms and thus reduce the duration of labor. ${ }^{6} \mathrm{HBB}$ has been used to shorten the duration of labor in several hospitals in Saudi Arabia and elsewhere in the world..$^{7-16}$ The mechanism by which it acts in the context of labor has not yet been elucidated, and evidence of its efficacy has been largely anecdotal.

Major studies have been carried out to evaluate the effects of the injectable or suppository form of $\mathrm{HBB}$ on cervical dilatation; a majority of these studies demonstrated the efficacy of HBB in augmenting labor. ${ }^{7-16}$ However, a few studies showed no effect of HBB on accelerating labor. ${ }^{15}$ In addition, some studies also used oxytocin in active management of labor, which would certainly affect the duration of labor and increase bias in the results. Including both primiparous and multiparous patients can also affect the results, because the duration of labor is shorter in multiparous women, even without any method of augmentation. Therefore, in our study, we enrolled only primigravid women in spontaneous labor with no other means of augmentation.

The specific objectives of this study were to assess whether $\mathrm{HBB}$ is effective in hastening cervical effacement and dilatation, thus shortening the duration of the first stage of labor in primiparous women without labor augmentation. We also intended to determine whether the use of HBB in the first stage of labor has any associated increases in complications, such as an increase in blood loss or the rate of cesarean deliveries, or a decrease in neonatal Apgar scores.

\section{Methods}

The study was designed as a double blind, randomized, controlled clinical trial comparing two groups of women: one group received $\mathrm{HBB}$, while the other (control) group received a placebo. Enrollment for this study commenced in January 2009 and was completed in January 2010.

Approval of this study was obtained prior to its onset from the Ethical Committee of the University of Dammam. Women were recruited from the delivery room of our institution, and full informed consent was obtained. The consent form was signed by the patient after explaining the purpose of enrollment in the study, aimed at shortening the first stage of labor using HBB, and describing potential side effects. Adequate opportunity was provided for the women to voice any questions or concerns regarding the study.

The sample size was based on a pilot study of ten patients, which determined a mean duration of the first stage of labor of 220 minutes. A reduction of 55 minutes in the first stage of labor was determined to be clinically significant. A sample size of 88 patients in both groups was determined to be required to detect this difference, with a type I error of 0.05 and a type II error of 0.20 , to compensate for drop out cases and adjust for normality. A total of 110 women were initially enrolled.

Women included in the study were all 17 years or older, primiparous, had singleton pregnancies, had vertex presentation at term (gestational age between 37-41 weeks +6 days), and had no chronic or pregnancy-induced illnesses. None of the women had any contraindications to vaginal delivery, and all were in established, spontaneous labor with either intact membranes or spontaneous rupture of membranes for less than 12 hours. Exclusion criteria included previous uterine scarring, malpresentation, antepartum hemorrhage, multiparity, twin pregnancy, induced delivery, any medical disease, oxytocin induction, prolonged premature rupture of the membrane (more than 12 hours), and epidural analgesia. Established labor was defined as the presence of regular uterine contractions (2-3 per 10 minutes) associated with progressive cervical effacement and dilatation.

Randomization was accomplished using cards. One hundred and twenty sequentially numbered, opaque, sealed envelopes were used containing 60 cards labeled as placebo and 60 cards labeled as HBB (as Buscopan ${ }^{\circledR}$, Boehringer Ingelheim GmbH, Germany). All of the envelopes were mixed together randomly and placed in a box.

Once the patient was admitted in active labor, a history and physical examination was conducted by the attending physician. A partogram was maintained throughout labor and vaginal examinations were conducted and recorded every 2 hours. The drug was given only when the cervix was fully effaced and was dilated to $3-4 \mathrm{~cm}$. The attending nurse was asked to complete a form detailing the duration of labor during the first, second, and third stages, the mode of delivery, maternal complications, and neonatal conditions at birth (Apgar score and weight).

After the physician prescribed HBB, the nurse attending the patient informed the nurse in charge of the shift, who selected a random card from the box; only she was aware of the contents of the syringe. Sterile syringes were prepared 
by the nurse in charge according to the label on the card, without disclosing its contents to the staff or physician. She then enclosed the card inside the envelope and affixed it to the patient's partogram after delivery. Each syringe contained either $2 \mathrm{~mL} \mathrm{HBB}$ (40 mg) or $2 \mathrm{~mL}$ normal saline. Both liquids are colorless; thus, the syringes containing the drug were indistinguishable from those containing the placebo. Participants received the contents of the syringe as a single dose, given intramuscularly (IM). The patient and the caregivers were blinded as to whether the active drug or placebo was being administered. Routine amniotomy was performed for all women in established labor who were found to have cervical dilatation of $\geq 4 \mathrm{~cm}$, and who had not had spontaneous rupture of membranes. Opioid analgesia was routinely given after the amniotomy (meperidine hydrochloride $100 \mathrm{mg}$ IM and promethazine $25 \mathrm{mg}$ IM).

Laboring mothers were monitored in bed, and continuous electronic cardiotocography was conducted to monitor fetal wellbeing. The duration of the first stage was calculated from the time of cervical dilatation of $3-4 \mathrm{~cm}$ in active labor until a fully dilated cervix was observed upon vaginal examination conducted every 2 hours. Oxytocin augmentation was initiated if the initial progress of labor (as assessed through partographs) was unsatisfactory. These patients were excluded from the study. Intervention by instrumentation or cesarean delivery was dictated by the usual obstetric indications.

All data sheets (containing the raw data obtained during the study) were collected by the first investigator. The nurse in charge communicated directly with the first investigator via the assigned mobile telephone, available in the delivery room, for any needed clarifications.

Normally distributed data were expressed as means \pm standard deviations (SDs), and noncontinuous data, such as cervical dilation, was expressed as a median and interquartile range. Data were tested for a normal distribution using the Kolmogorov-Smirnov test. Categorical data such as instrumental delivery, cesarean section, postpartum hemorrhage, and perineal tear were analyzed using the chi-square test or Fisher's exact test, as appropriate. An unpaired $t$-test was used for continuous data and the Mann-Whitney $U$ test (the nonparametric equivalent) was used for comparison of noncontinuous data. $P<0.05$ was considered significant. Statistical analyses were performed using statistical software version 7 for Windows (StatSoft Inc, Tulsa, OK, USA).

The duration of the first, second, and third stages of labor were right-skewed on initial analysis. The values were natural $\log$ transformed to attain normalization; this normalization was subsequently confirmed using the onesample Kolmogorov-Smirnov test. The independent sample $t$-test and Mann-Whitney $\mathrm{U}$ test were then used to evaluate the significance of mean and median differences for both raw and transformed groups of data. Outliers were noted in both the blood loss data and Apgar scores; analyses with and without these outliers did not change the results.

\section{Results}

A total of 110 women consented to be enrolled in the study; however, 13 women met the exclusion criteria, such as a patient request for epidural analgesia or the need for oxytocin in the first stage of labor. Thus, data for 97 women were ultimately included for analysis. Of these, 45 women received the placebo and 52 received $\mathrm{HBB}$.

Patients in both groups were 17-39 years old, with a median of 23.9 years in the HBB group and 24.4 years in the control group. The mean height and weight in the HBB group were $155.5 \mathrm{~cm}$ and $68.1 \mathrm{~kg}$, respectively, and in the control group were $154.2 \mathrm{~cm}$ and $66.1 \mathrm{~kg}$, respectively. The median gestational age was 39 weeks +4 days in both the HBB and control groups. The two groups were comparable in cervical findings at the time of presentation and recruitment into the study, ie, all patients were $3-5 \mathrm{~cm}$ dilated with a median of $3 \mathrm{~cm}$ in both groups, with strong uterine contractions (Table 1).

The mean duration of the first stage in the control group was 215 minutes, compared with 165 minutes in the HBB group (Figure 1), with a difference of 50 minutes. This represents a statistically significant decrease of $23.3 \%(P=0.001 ; 95 \% \mathrm{CI}$ $32-115)$. The mean duration of the combined first and second stages of labor was also calculated and showed a statistically

Table I Patient characteristics and incidence of labor

\begin{tabular}{llll}
\hline Characteristic & $\begin{array}{l}\text { Control } \\
\text { group } \\
(\mathbf{n}=\mathbf{4 5})\end{array}$ & $\begin{array}{l}\text { Hyoscine } \\
\text { butylbromide } \\
\text { group } \\
(\mathbf{n}=\mathbf{5 2})\end{array}$ & $\mathbf{P}$ \\
\hline Age (years) & $24.4(5.36)$ & $23.9(4.04)$ & 0.638 \\
Height (cm) & $154.2(10.38)$ & $155.5(5.6 \mathrm{I})$ & 0.762 \\
Weight (kg) & $66 . I(\mathrm{I} 2.93)$ & $68.1(\mathrm{II} .95)$ & 0.458 \\
$\begin{array}{l}\text { Gestational age (weeks) } \\
\text { Cervical dilatation (cm) }\end{array}$ & $39.4(\mathrm{I} . \mathrm{II})$ & $39.4(0.98)$ & 0.683 \\
$\begin{array}{l}\text { Spontaneous rupture } \\
\text { of the membrane }\end{array}$ & $10 / 35$ & $3(3-4)$ & 0.596 \\
(yes/no) & & $23 / 29$ & $0.039 *$ \\
\hline
\end{tabular}

Notes: For patient characteristics, data are expressed as means (SD - standard deviation); for cervical dilation, data are median and interquartile range; for spontaneous rupture of the membrane, data are numbers. *Significant difference between the two groups. 


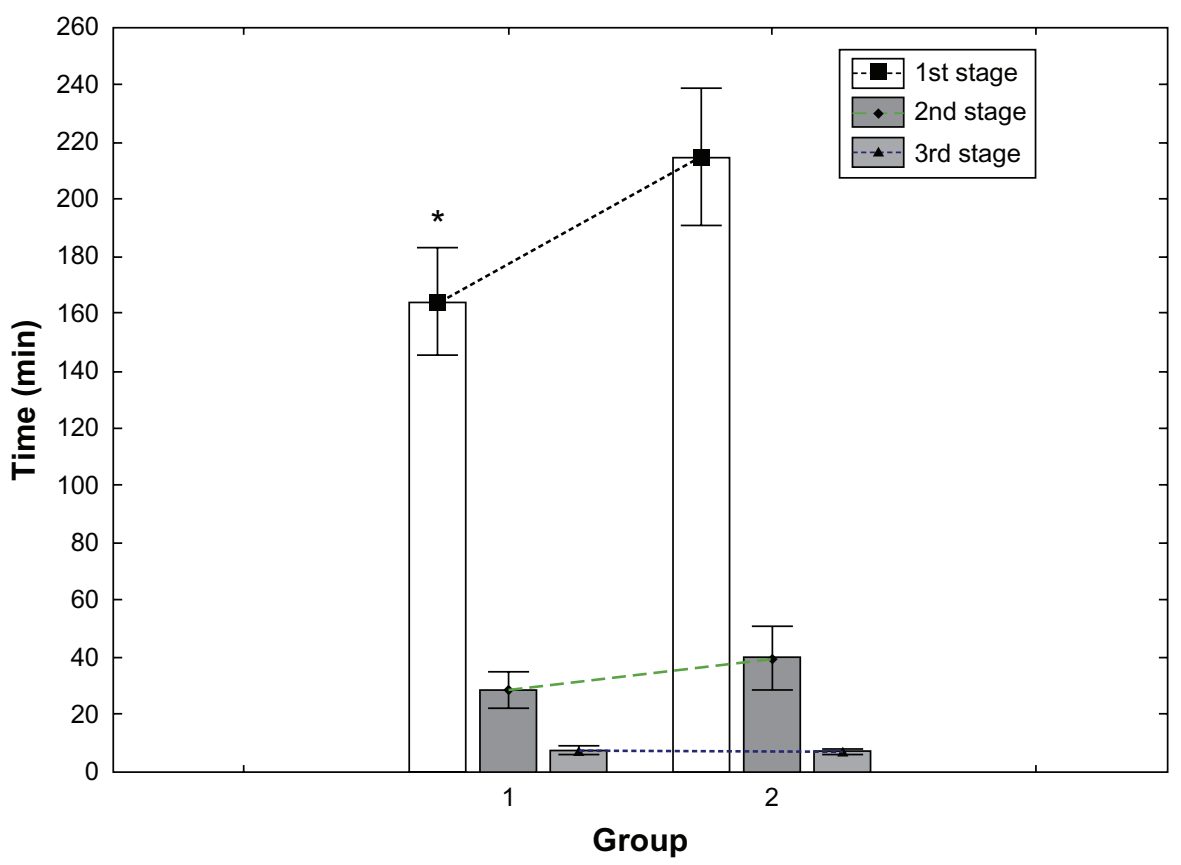

Figure I Mean durations of the first, second, and third stages of labor in the control group (group 2) and the hyoscine butylbromide group (group I). Notes: Vertical bars denote $0.95 \%$ confidence intervals. $* P<0.00$ I indicates a significant difference between the hyoscine butylbromide and control groups.

significant difference $(P<0.001)$, with a mean duration of 251 minutes (92 SD [standard deviation]) in the control group and 190 minutes (75 SD) in the HBB group, indicating a 24\% reduction in the duration of labor from a $4 \mathrm{~cm}$ dilated cervix until full expulsion of the fetus (Table 2). There were no significant changes in the durations of the second $(P=0.063)$ or third $(P=0.0418)$ stages of labor.

There were no significant differences in the cardiotocographic findings between the study and control group. There was no difference in associated blood loss between the groups; all of the data fell within an average range, with no cases of postpartum hemorrhage.

There was a slightly higher rate of instrumental delivery $(15.3 \%, \mathrm{n}=6)$ in the control group versus $13 \%(\mathrm{n}=6)$ in the HBB group; however, the difference was not statistically significant. In addition, there was no significant increase in the cesarean rate among the women receiving HBB (9\%) compared with the control group $(6 \% ; P=0.721)$. There were three cesareans in the control group, one case due to failure to progress and two cases due to fetal distress, while in the HBB group there were five cases, two due to failure to progress and three due to fetal distress (Table 3).

Only 22 patients (40\%) in the HBB group required opioid analgesia, while all of those in the control group did, a statistically significant indication of the efficacy of HBB as an analgesic in addition to its role in augmentation of labor $(P<0.001 ;$ Table 4$)$.
There were no significant differences between the Apgar scores recorded at 1 and 5 minutes between the groups. Of 97 neonates, five babies in the study group (including three neonates by cesarean) and eight babies in the control group (including two neonates by cesarean) had Apgar scores of 7-8 at 1 minute. These babies were held in a nursery under observation for 24 hours and were discharged in good condition. The remaining 84 neonates had a score of 9 at 1 minute. The Apgar score was $8-10$ at 5 minutes for all 97 babies, with all babies surviving. The drug was well tolerated by all neonates and no adverse effects were noted in either group $(P=0.380)$.

\section{Discussion}

$\mathrm{HBB}$, in the form of Buscopan, has been used to shorten the duration of labor in several hospitals in Saudi Arabia. Interestingly, it was introduced and suggested by midwives

Table 2 Mean duration of first and second stage of labor

\begin{tabular}{llll}
\hline Stage of labor & $\begin{array}{l}\text { Control } \\
\text { group } \\
(\mathbf{n}=\mathbf{4 5})\end{array}$ & $\begin{array}{l}\text { Hyoscine } \\
\text { butylbromide } \\
\text { group } \\
(\mathbf{n}=\mathbf{5 2})\end{array}$ & $\boldsymbol{P}$ \\
\hline First stage (minutes) & $214(79)$ & $165(67)$ & $<0.001$ \\
Second stage (minutes) & $40(34)$ & $28(20)$ & 0.063 \\
First + second stage & $251(92)$ & $190(75)$ & $<0.001$ \\
(minutes) & & & \\
\hline
\end{tabular}

Note: Data are expressed as means (SD - standard deviation). 
Table 3 Outcome of delivery

\begin{tabular}{llll}
\hline Outcome & $\begin{array}{l}\text { Control } \\
\text { group } \\
\mathbf{( n = 4 5 )}\end{array}$ & $\begin{array}{l}\text { Hyoscine } \\
\text { butylbromide } \\
\text { group } \\
(\mathbf{n}=\mathbf{5 2})\end{array}$ & $\mathbf{P}$ \\
\hline $\begin{array}{l}\text { Instrumental delivery } \\
\text { (yes/no) }\end{array}$ & $6 / 39$ & $6 / 46$ & 0.967 \\
$\begin{array}{l}\text { Cesarean section } \\
\text { (yes/no) }\end{array}$ & $3 / 42$ & $5 / 47$ & 0.721 \\
$\begin{array}{l}\text { Postpartum hemorrhage } \\
\text { (yes/no) }\end{array}$ & $0 / 45$ & $0 / 52$ & 0.000 \\
$\begin{array}{l}\text { Tear (yes/no) } \\
\text { Apgar score (9-10 vs <9) }\end{array}$ & $0 / 45$ & $2 / 50$ & 0.497 \\
\hline
\end{tabular}

trained in India and the Philippines. It is used as an intravenous or intramuscular injection for active management of labor, to shorten labor and to overcome an edematous cervix. It is typically given with promethazine to sedate the patient to some degree and eliminate the need for further analgesia. Whereas its analgesic properties are likely negligible in the context of labor, its value lies in shortening the duration of the first stage of labor. Consequently, the overall time spent in pain by the laboring mother is reduced. Although its use in some hospitals in Saudi Arabia is fairly widespread, evidence for its efficacy in this population has been largely anecdotal.

A review of the literature produced several older and some recent studies relating to HBB and its use in labor. Few were randomized, double-blind studies, and none was conducted in an Arabian population similar to that of Saudi Arabia. Most of these studies have used the intramuscular route, ${ }^{7,9,10}$ with a few isolated studies using intravenous ${ }^{11,13}$ and suppository routes, ${ }^{12}$ including varying doses of 40 or $20 \mathrm{mg}$ as a single or repeated dose. The results of this study support previous reports that HBB shortens the first stage of labor.

The primary strength of this study, in addition to being a randomized double blind controlled trail, is that it was limited to primigravid patients in spontaneous labor with the exclusion of patients receiving oxytocin and epidural analgesia from the final statistical analysis, as these factors may shorten or prolong labor, respectively.

The results of our study are important for several reasons. Potential benefits of reduced first stage duration include reduced duration of severe pain, incidence of chorioamnionitis, neonatal sepsis, and puerperal sepsis, all of which increase in women with prolonged labor. A reduced need for repeat doses of opioid analgesia, which is associated with neonatal respiratory depression, is also a substantial benefit of a shorter labor process. This is particularly true in regions where epidural analgesia is not widely available, and
Table 4 Need for analgesia

\begin{tabular}{llll}
\hline Analgesia & $\begin{array}{l}\text { Control } \\
\text { group } \\
(\mathbf{n}=\mathbf{4 5})\end{array}$ & $\begin{array}{l}\text { Hyoscine } \\
\text { butylbromide } \\
\text { group } \\
(\mathbf{n}=\mathbf{5 2})\end{array}$ & $\mathbf{P}$ \\
\hline Meperidine & 45 & 22 & $<0.001$ \\
No meperidine & 0 & 30 & - \\
\hline
\end{tabular}

thus opioid medications are used with increased frequency to relieve labor pain in the first stage. Additionally, we are certain that any intervention that can safely reduce the amount of time spent in the painful process of parturition will be greatly appreciated by women.

The reduction in first stage duration may also prove to be of particular importance for women with a borderline placental reserve, oligohydramnios, or risk of prolonged variable deceleration during labor, as may be encountered in women with hypertension (both chronic and gestational) and in women with sickle cell anemia, which is quite common in our population. Prolonged labor in these women may result in the fetoplacental reserves becoming depleted, with consequent signs of fetal distress and an increased rate of cesarean.

Our study demonstrated that HBB significantly reduces the duration of the first stage of labor. However, we also demonstrated that there was no statistical difference in the durations of the second and third stages of labor (Figure 1), implying that HBB primarily acts on the cervix rather than promoting uterine activity. This is important, as it obviates the concern regarding an excessively rapid second stage, which can result in both maternal complications (such as increased risk of lacerations or vaginal hematoma, particularly in the primigravida) and neonatal complications (such as intracranial hemorrhage due to rapid, uncontrolled decompression of the fetal head at delivery). Of equal significance, the average blood loss at delivery was identical in both groups (Table 3). This suggests that there is no adverse effect on the contractile ability of the uterus in the postpartum period, as might theoretically be anticipated based on its antiparasympathetic pharmacological activity.

The difference in the duration of the first stage of labor between the two groups in the trial was 50 minutes. However, the women were subjected to examinations at regular 2 hour intervals; thus, the error in measuring the length of the first stage may have been as much as $\pm 1- \pm 2$ hours, rendering the clinical significance of the result marginal. To assess this possibility, we repeated the analysis using the combined first and second stage duration, and the results remained nearly the same, demonstrating a statistically significant difference 
in duration between the HBB and placebo groups, with a mean 64 minute difference. Thus, any error in measurement of the first stage time was not contributory to the outcome of the study.

We did not attain the numbers we had initially aimed for due to the elimination of 13 women. However, even using a sample size of almost 50 women per arm of the study, we still achieved a power of over $90 \%$. Another limitation in the study design was that patients' acceptance was not assessed, a parameter that is as important as efficacy.

The difference in average Apgar scores for the infants in the two groups was not statistically significant (Table 3). The number of neonates with a lower Apgar score was greater in the control group, which is likely related to the use of opioids in $100 \%$ of these patients, causing mild respiratory depression in the neonate and a reduction in the Apgar score. This suggests that there were no clinically significant effects of $\mathrm{HBB}$ on the neonates for any of the major organ systems. Although the study was not sufficiently powered for absolute exclusion of fetal/ neonatal adverse effects, the initial examination of each infant, on which the Apgar scores were based, showed no discernible differences in the infants in the HBB group compared with the control group. There was also no difference in the incidence or severity of cardiotocographic changes intra-partum (decreased beat-to-beat variability and fetal tachycardia are potential adverse effects of HBB) or of neonatal tachycardia.

Hyoscine/scopolamine has an excellent safety profile and as such has been approved by the Food and Drug Administration in the USA for preoperative use in women scheduled for cesarean section. In addition, the drug is considered compatible with breastfeeding. ${ }^{11}$ We believe that the evidence from our study, taken in the context of other available evidence based information, justifies our assessment that the drug is safe and that there are no adverse effects associated with its use in this context.

\section{Conclusion}

Based on the results of our study, and noting the supportive data from similar clinical trials, we conclude that $\mathrm{HBB}$ is effective in significantly reducing the duration of the first stage of labor and reducing the need for further analgesia, and that it is not associated with any apparent adverse outcomes in the mother or neonate. Further long term evaluation will be necessary to fully evaluate the scope of benefits that this reduction may confer.

\section{Disclosure}

We certify that no actual or potential conflicts of interest in relation to this article exist.

\section{References}

1. Lopez-Zeno JA, Peaceman AM, Adashek JA, Socol ML. A controlled trial of a program for the active management of labour. $N$ Engl J Med. 1992;326:450-454.

2. Frigoletto FD Jr, Lieberman E, Lang JM, et al. A clinical trial of active management of labour. $N$ Engl J Med. 1995;333:745-750.

3. Sadler LC, Davison T, McCowan LM. A randomised controlled trial and meta-analysis of active management of labour. BJOG. 2000;107(7): 909-915.

4. Hotwani J, Ainapure SS. Hyoscine butylbromide suppositories. Indian Medical Gaz. 2000:217-219.

5. Corsen G. A study of the use and mode of action of the antispasmodic drug Buscopan in gynecology and obstetrics. Med Klin. 1983;48: 2186-2188.

6. Philpott RH, Castle WM. Cervicographs in the management of labor in primigravidae: The alert line for detecting abnormal labour. J Obstet Gynecol Br Commonw. 1972;79(7):592-598.

7. Bhattacharya P, Joshi SG. Acceleration of labour with intramuscular Buscopan injection. J Obstet Gynecol Ind. 1985:1014-1017.

8. Blasko ST, Demeter J. The effect of intramuscularly administered drotaverine on the dilatation stage of uncomplicated deliveries. Obstet Gynecol Today. 1998;3:723-737.

9. Baracho HM, Kamat JR, Kunalhekar JI. Buscopan in acceleration of labor. J Obstet Gynecol Ind. 1984;34:509-512.

10. Samal S, Gupta U, Wable M. Buscopan (hyoscine-N-butylbromide) in acceleration of labour. Ind Med Gaz. 1998;82:8-10.

11. Sirohiwal D, Dahiya K, De M. Efficacy of hyoscine-N-butylbromide (Buscopan) suppositories as a cervical spasmolytic agent. Aust $N Z J$ Obstet Gynaecol. 2005;45(2):128-129.

12. Tewari K, Jabeen R, Sabzposh MA, Rabbani T. Comparison of hyoscinebutylbromide and valethamate bromide in shortening the duration of labor. Indian Med Gaz. 2003;137:15-19.

13. Samuels LA, Christie L, Roberts-Gittens B, Fletcher H, Frederick J. The effect of hyoscine butylbromide on first stage of labour in term pregnancies. BJOG. 2007;114(12):1542-1546.

14. Chan DPC. The use of Buscopan during the first stage of labor. Hong Kong Med Ass. CME Bull 1963;15:69-71.

15. Gupta B, Nellore V, Mittal S. Drotaverine hydrochloride versus hyoscine-N-butylbromide in augmentation of labor. Int $J$ Gynaecol Obstet. 2008;100(3):244-247.

16. Aggarwal P, Zutshi V, Batra S. Role of hyoscine N-butyl bromide (HBB, buscopan $^{\circledR}$ ) as labor analgesic. Indian J Med Sci. 2008;62:179-184.

Therapeutics and Clinical Risk Management

\section{Publish your work in this journal}

Therapeutics and Clinical Risk Management is an international, peerreviewed journal of clinical therapeutics and risk management, focusing on concise rapid reporting of clinical studies in all therapeutic areas, outcomes, safety, and programs for the effective, safe, and sustained use of medicines. This journal is indexed on PubMed Central, CAS,

Submit your manuscript here: http://www.dovepress.com/therapeutics-and-clinical-risk-management-journal

EMBase, Scopus and the Elsevier Bibliographic databases. The manuscript management system is completely online and includes a very quick and fair peer-review system, which is all easy to use. Visit $\mathrm{http}: / / \mathrm{www}$.dovepress.com/testimonials.php to read real quotes from published authors. 